\title{
ORIGINAL ARTICLE \\ Effects of a visuotemporal cue on walking ability of independent ambulatory subjects with spinal cord injury as compared with healthy subjects
}

\author{
N Pramodhyakul ${ }^{1,2}$, P Amatachaya ${ }^{2,3}$, T Sooknuan ${ }^{4}$, P Arayawichanon ${ }^{2,5}$ and S Amatachaya ${ }^{1,2}$
}

\begin{abstract}
Study design: An experimental, cross-sectional study.
Objectives: To investigate effects of using a visuotemporal cue on the walking ability of independent ambulatory subjects with spinal cord injury $(\mathrm{SCl})$ as compared with healthy subjects.
\end{abstract}

Setting: A tertiary rehabilitation center, Thailand.

Methods: Forty independent ambulatory subjects with $\mathrm{SCl}$ and healthy subjects participated in the study (20 subjects per group). All of them were assessed for their walking speed, stride length, cadence and percents of step symmetry under two conditions, including walking at their fastest speed with and without a visuotemporal cue along a $10 \mathrm{~m}$ walkway.

Results: When walking with a visuotemporal cue, walking speed, stride length and cadence of the subjects were significantly increased from the uncued condition $(P<0.01)$. However, the healthy subjects showed a significantly greater increase in walking speed and cadence, whereas, subjects with $\mathrm{SCl}$ demonstrated significantly higher improvement in stride length as compared with the other group $(P<0.001)$. In addition, the effects of the visuotemporal cue were significantly greater in those with less severity of $\mathrm{SCl}$ $(P<0.01)$.

Conclusion: The findings confirm the benefits of using a visuotemporal cue to improve variables relating to walking ability in subjects with intact integrative capability of the brain but with different levels of sensorimotor deterioration. The findings suggest the use of a visuotemporal cue to improve the effectiveness of programs in sport and exercise sciences, and rehabilitation treatments.

Spinal Cord (2014) 52, 220-224; doi:10.1038/sc.2013.160; published online 7 January 2014

Keywords: tetraplegia; paraplegia; external information; feedback; rehabilitation

\section{INTRODUCTION}

Most ambulatory patients with spinal cord injury (SCI) can walk nonfunctionally, which is commonly characterized by a slow walking speed, short and asymmetrical step length, and low walking cadence that attribute negative impacts to the ability of independent walking. ${ }^{1-5}$ Among several variables relating to walking ability, the increase in walking speed is crucial because it is likely accompanied by an increase in lower-extremity force production, distance walk and quality of walking (that is, step rhythm, foot placement, step symmetry and weight shift). ${ }^{4,5} \mathrm{An}$ increase in walking speed of at least $0.3 \mathrm{~m} \mathrm{~s}^{-1}$ was associated with ambulatory advancement in subjects with SCI. ${ }^{3}$ Zorner et al. ${ }^{6}$ also reported that individuals with SCI need a walking speed of at least $0.6 \mathrm{~m} \mathrm{~s}^{-1}$ to safely cross a street.

Previously, external cues, that is, visual, auditory and visuotemporal cues, have been successfully applied to facilitate movement initiation and continuation in patients with brain disorders, that is, stroke and Parkinson's disease. ${ }^{7-9}$ These cues provide extrinsic feedback supplementary to the deprivation of internal regulation of movement control that subsequently improves variables related to walking ability of these individuals. ${ }^{8,9}$ However, patients with SCI have intact integrative capability of the brain but they are unable to fully perceive somatosensory information, which normally acts as an internal reference of movement correction and has a vital role in the ability of movement control. ${ }^{4,10}$ This impairment reduces ability of the patients to modify and generate optimal movements according to the demands of everyday activities. ${ }^{4}$

Nonetheless, the human movement systems are very flexible that allows the utilization of supplementation from other relating systems. ${ }^{11}$ The previous studies ${ }^{4,12}$ found that the application of an alternative source of inputs, namely, the visuotemporal cue, helped ambulatory subjects with SCI to improve their walking ability. However, the studies were executed in a single group of subjects, so the findings may not ensure the effects of external information in individuals with intact brain functions but with some degrees of sensorimotor deterioration. The data comparison with those who have normal brain functions and no sensorimotor impairment would further explain the benefit of visuotemporal cue in these individuals. Therefore, this study assessed the effects of using a visuotemporal cue on the walking ability of independent ambulatory subjects with SCI as compared with healthy subjects. To ensure the influence of the cue in

${ }^{1}$ School of Physical Therapy, Faculty of Associated Medical Sciences, Khon Kaen University, Khon Kaen, Thailand; ${ }^{2}$ Improvement of Physical Performance and Quality of Life (IPQ) research group, Khon Kaen University, Khon Kaen, Thailand; ${ }^{3}$ Department of Mechanical Engineering, Faculty of Engineering and Architecture, Rajamangala University of Technology Isan, Nakhon Ratchasima, Thailand; ${ }^{4}$ Department of Electronics Engineering, Faculty of Engineering and Architecture, Rajamangala University of Technology Isan, Nakhon Ratchasima, Thailand and ${ }^{5}$ Department of Rehabilitation Medicine, Faculty of Medicine, Khon Kaen University, Khon Kaen, Thailand

Correspondence: Associate Professor S Amatachaya, School of Physical Therapy, Faculty of Associated Medical Sciences, Khon Kaen University, Khon Kaen 40002, Thailand. E-mail: samata@kku.ac.th

Received 19 September 2013; revised 17 November 2013; accepted 1 December 2013; published online 7 January 2014 
individuals with different degrees of sensorimotor deterioration, the data in subjects with SCI were also analyzed according to the severity of SCI. The researchers hypothesized that the Supplementary Information provided by a visuotemporal cue would assist subjects in both groups to walk at a greater speed than when they walked at their own determination without using the cue. However, the sensorimotor impairments may limit the ability of subjects with SCI to modify their movements in response to the external information provided. Therefore, the effects of external cues in subjects with SCI, particularly those with severe SCI, would be less than those in healthy subjects.

\section{MATERIALS AND METHODS}

\section{Subjects}

The researchers recruited patients with motor-incomplete SCI (the American Spinal Injury Association (ASIA) Impairment Scale: AIS C and D) who were able to walk independently with or without a walking device for at least $10 \mathrm{~m}$, aged at least 18 years, from a major tertiary referral hospital, Thailand. The exclusion criteria were any signs or symptoms that might affect ambulatory ability such as severe spasticity of the lower-extremity muscles (the modified Ashworth scale $>2$ ), pain in the muscles or joints with an intensity of pain more than 5 out of 10 on a numeric rating pain scale, deformities of the spine and lower extremities (that is, scoliosis, kyphosis and equinovarus). Furthermore, the researchers invited healthy subjects who did not have any conditions that might affect walking ability, matched by gender and age ( \pm 5 years), compared with those with SCI. A sample size calculation for two group comparisons using data from a pilot study indicated that the study needed 10 , 20, 14 and 351 subjects per group for the four parameters measured in the study including walking speed, stride length, cadence and percents of step symmetry, respectively. On the basis of the availability of the patients according to the criteria used in the study, the researchers recruited 20 subjects per group. All of them provided a written informed consent document approved by the Khon Kaen University Ethics Committee for Human Research before participation in the study.

\section{Experimental protocol}

Subjects were interviewed and assessed for their baseline demographics, SCI characteristics and baseline walking ability. After sufficient rest, subjects were assessed for their walking ability under the two conditions. Details of the test conditions are as follows.

Walking without the visuotemporal cue (uncued condition). This condition served as a baseline condition in which subjects needed to walk at their fastest and safe speed along a $10 \mathrm{~m}$ straight walkway without any cues.

Walking with the visuotemporal cue condition. The test required a visuotemporal cue machine, which consisted of a countdown timer, electronic light bars and laser sensors (Figure 1a) that was positioned at the end of the walkway (Figure 1b), to provide temporal information for the subjects to perceive visually. The countdown timer was set at $25 \%$ less than the time required for the uncued condition. . $, 8,12^{2}$ On start, the light bars gradually decreased according to the remaining time, and the subjects were required to walk at their fastest speed along a $10 \mathrm{~m}$ straight walkway to the end of the walkway before the running-out of the last light bar (Figure 1b). If the subjects were able to successfully walk through the laser beam on time, the circuit was interrupted and no alarm was on. In contrast, if the time ran out before the subjects could walk through the beam, the visuotemporal cue machine gave an alarm.

During the test, a bright-yellow-colored tape was placed on the floor in order to guide the subjects to walk along a straight walkway (Figure 1b). Subjects performed five trials per condition in which the first two trials served as familiarization sessions (practice trials), and the other three trials were used for data recording. ${ }^{4,8,12}$ Subjects with SCI were allowed to use a customary walking device. All of them had to fasten a lightweight safety belt and had an assessor walking alongside throughout the test to ensure safety (Figure 1b).

\section{a}

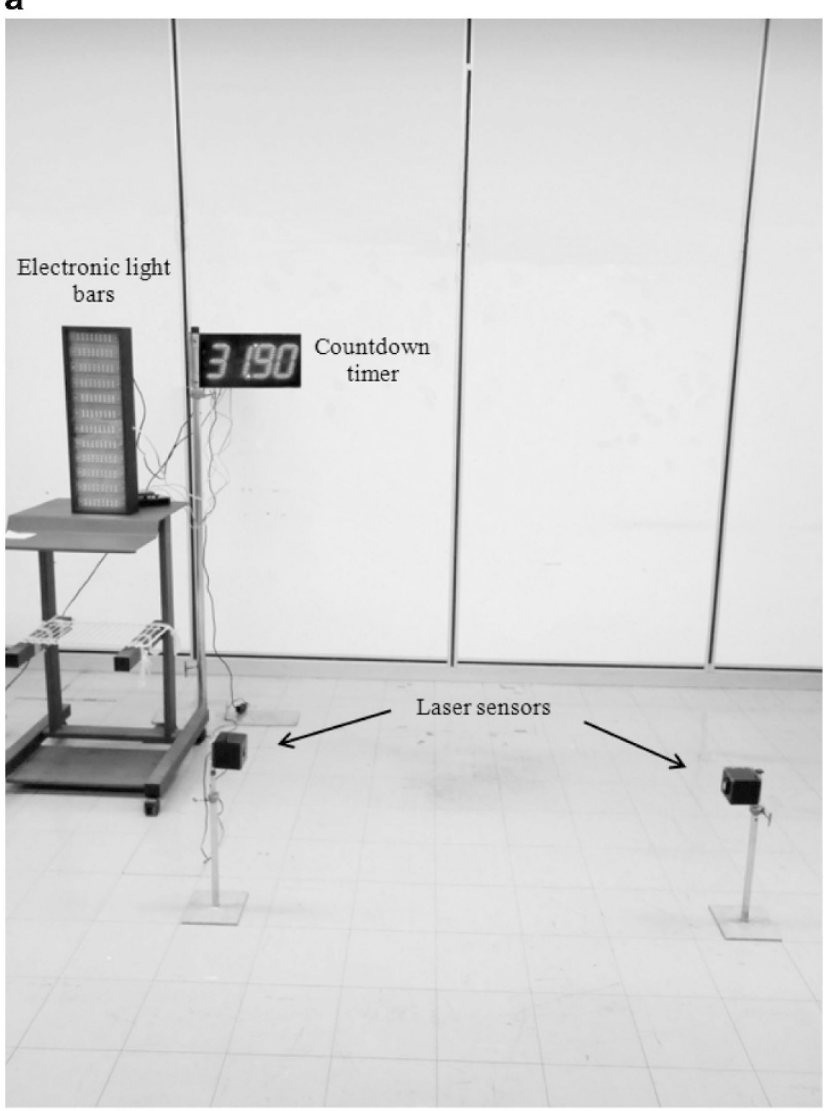

b

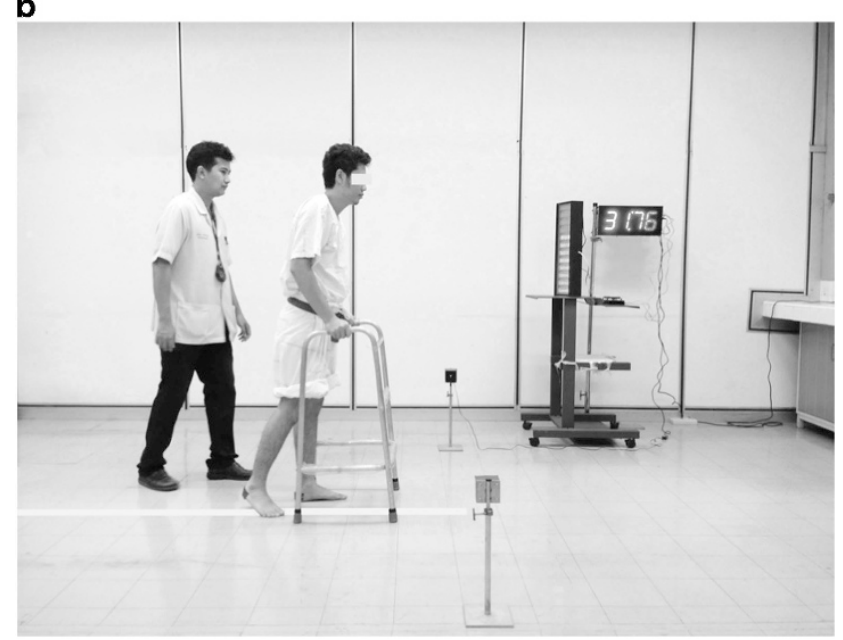

Figure 1 The visuotemporal cue condition (a) components of the visuotemporal cue machine, (b) a subject walking with the visuotemporal cue.

Subjects did not receive any other encouragement during the tests and were able to take a period of rest between the trials as required.

\section{Outcome measures}

Subjects' walking speed, stride length, cadence and percents of step symmetry using a digital camera with a frame rate of $60 \mathrm{~Hz}$ and the method of manual digitization as proposed by Said et al. ${ }^{13}$ were measured. The method had high validity compared with the PEAK motion analysis $\left(\mathrm{ICC}_{3,1}=0.95-0.99\right)$ and high reliability $\left(\mathrm{ICC}_{3,1}=0.94\right)$, and could be executed in a rehabilitation ward. 
Before the test, the video recording system was positioned perpendicular to the walkway, in line with the middle of the walkway to capture a $3 \mathrm{~m}$ section of the walkway. Then the system was calibrated using a $60 \mathrm{~cm}$ object. A step length was measured by counting the horizontal pixels between the heel of one foot and the heel of the other foot, and scaling with respect to the calibration object. The total distance of the right and left step lengths represented a stride length. ${ }^{13}$ The cadence was measured by counting the number of steps and time used over the middle $3 \mathrm{~m}$ of the walkway and converting to the number of steps within $60 \mathrm{~s}$ (steps per min).,12 Then, walking speed was calculated using the formula: $\left[\frac{\text { stride length } \times \text { cadence }}{120}\right] .14$ Furthermore, percents of step length symmetry were computed using the formula: $\left[\frac{\text { average step length of the more affected leg }}{\text { average step length of the less affected leg }} \times 100\right] .4,12$

\section{Statistical analyses}

Descriptive statistics were applied to explain baseline demographics of the subjects and findings of the study. The differences of walking variables between the uncued and visuotemporal cue conditions within the groups were compared using the dependent-sample $t$-test. Then, the independent-samples $t$-test was utilized to compare the changes in walking variables between the groups. The level of statistical significance was set at $P<0.05$.

\section{RESULTS}

Forty subjects participated in the study ( 20 subjects per group and 18 males in each group). For subjects with SCI, most of them were at a chronic stage $(n=16)$ and required a walking device (13 subjects used a standard walker and 1 subject walked with axillary crutches). Their baseline demographics were not significantly different between the groups $(P>0.05$, Table 1$)$.

While using the visuotemporal cue, every subject could walk to the end of the walkway on time or nearly. Their walking speed, stride length and cadence were significantly increased from the baseline walking ability $(P<0.01$, Table 2$)$. The improvement in walking speed and cadence of healthy subjects was significantly greater than that of SCI subjects, whereas, the increment in stride length of subjects with SCI was significantly greater than that in healthy subjects $(P<0.001$, Table 2).

In subjects with AIS C and D, their walking speed, stride length and cadence were also significantly increased from the baseline ability (uncued condition), particularly in those with AIS D $(P<0.01$, Table 3). Nevertheless, only the changes in walking speed and cadence of subjects with AIS D were significantly different from those with AIS $\mathrm{C}(P<0.01)$, but not for the stride length and percents of step symmetry $(P>0.05$, Table 3$)$.

\section{DISCUSSION}

Adequate walking speed is an important determinant for the ability to perform activities in daily living and community participation. ${ }^{2}$ Apart from a comfortable speed, Van Hedel et al. ${ }^{15}$ indicated that the ability

Table 1 Baseline demographics of subjects with incomplete spinal cord injury (iSCl) and healthy subjects

\begin{tabular}{lccc}
\hline Variable & $\begin{array}{c}\text { Subjects with iSCl } \\
(\mathrm{n}=20)\end{array}$ & $\begin{array}{c}\text { Healthy subjects } \\
(\mathrm{n}=20)\end{array}$ & $\begin{array}{c}\text { P- } \\
\text { value }^{\text {a }}\end{array}$ \\
\hline Age (years) & $42.00 \pm 12.82$ & $41.65 \pm 12.72$ & 0.931 \\
Height (m) & $166.05 \pm 3.98$ & $167.05 \pm 4.11$ & 0.439 \\
Weight (kg) & $61.64 \pm 9.76$ & $62.65 \pm 7.12$ & 0.711 \\
Body mass index & $22.42 \pm 3.48$ & $22.45 \pm 2.43$ & 0.977 \\
$\left(\mathrm{~kg} \mathrm{~m}^{-2}\right)$ & & &
\end{tabular}

Note: the data are presented using mean \pm s.d.

a $P$-value from the independent-samples $t$-test with the level of significant difference at $P<0.05$. to increase walking speed may clearly reflect the ability to respond to community challenges. Therefore, the study investigated the fastest walking speed of subjects while walking with and without the visuotemporal cue. The findings indicated that the visuotemporal cue could facilitate the subjects to significantly improve their walking ability from the baseline (uncued) condition (Table 2). The amount of increment in healthy subjects was significantly greater than that in subjects with SCI but only for walking speed and cadence. On the contrary, subjects with SCI demonstrated significantly higher improvement in stride length than the healthy subjects $(P<0.001$, Table 2). When comparing the findings in subjects with SCI who had different degrees of severity, the benefit of the visuotemporal cue was significantly greater in those with less severity of the lesions (AIS D) (Table 3).

Beek and van Wieringen ${ }^{10}$ reported that movements or actions are strongly related to perceptual information, in which all actions are forcefully influenced by surrounding information as picked up by the perceptual system. Information is specific where the nature of the information helps individuals to determine the level of force generation for the action. ${ }^{16}$ The visuotemporal cue provided temporal information that facilitated subjects to modify their movements in order to successfully accomplish the task. Therefore, healthy subjects showed significant improvement of variables relating to walking ability (Table 2). For individuals with SCI, the utilization of external information provides extrinsic feedback supplementary to the reduction of intrinsic feedback that facilitates the intrinsic dynamics of the movement system through synchronizing or entraining the phase coupling among body segments. ${ }^{4,10,12}$ However, the sensorimotor deterioration following SCI limited their ability to modify the movements in response to the information given. Therefore, these subjects showed significantly less improvement than the healthy subjects (Table 2).

The increment in walking speed is normally achieved by increased cadence and/or stride length. ${ }^{14}$ In healthy subjects, it is commonly known that stride length increases linearly as walking speed increases, and cadence would be increased at a higher speed in order to help individuals to cover a certain distance in a certain amount of time. ${ }^{17}$ In other words, when the limitation of stride length is reached, increment in cadence is the only possible strategy to increase walking speed. ${ }^{18}$ As a result, healthy subjects lengthened their stride length to increase speed while walking without the external cue (uncued condition). Then, the increment in walking cadence was the only possible strategy by which they could further enhance their walking speed in response to the external information provided. In contrast, the sensorimotor deterioration due to SCI reduced the ability of balance control and selfconfidence while walking, thus the subjects likely walked with a short step length. ${ }^{1}$ The external information provided may draw their attention and increase motivation and, therefore, the subjects showed an obvious increase in stride length while walking with the visuotemporal cue (Table 2).

The results also demonstrated that the visuotemporal cue could help subjects with AIS D to increase variables relating to walking to a greater extent than those with AIS C (Table 3). Having AIS C implied that subjects were faced with severe sensorimotor impairments that highly limited their ability to modify or generate movement according to the demands of the task. Consequently, subjects with AIS C showed significantly less improvement than those with AIS D when using the visuotemporal cue (Table 3 ). The significant differences with high 
Table 2 Walking ability in subjects with incomplete spinal cord injury (iSCI) and healthy subjects when walking with and without a visuotemporal cue

\begin{tabular}{|c|c|c|c|c|c|c|c|c|c|}
\hline \multirow[t]{2}{*}{ Variable } & \multicolumn{3}{|c|}{ Subjects with iSCI $(\mathrm{n}=20)$} & \multicolumn{3}{|c|}{ Healthy subjects $(\mathrm{n}=20)$} & \multicolumn{3}{|c|}{ Mean difference ${ }^{a}$} \\
\hline & $\begin{array}{l}\text { Uncued } \\
\text { condition }\end{array}$ & $\begin{array}{c}\text { Visuotemporal cue } \\
\text { condition }\end{array}$ & $\mathrm{P}$-value & $\begin{array}{l}\text { Uncued } \\
\text { condition }\end{array}$ & $\begin{array}{c}\text { Visuotemporal cue } \\
\text { condition }\end{array}$ & $\mathrm{P}$-value & $\begin{array}{c}\text { Subjects with } \\
\text { iSCl }\end{array}$ & $\begin{array}{l}\text { Healthy } \\
\text { subjects }\end{array}$ & $\mathrm{P}$-value \\
\hline $\begin{array}{l}\text { Walking speed } \\
\left(\mathrm{ms}^{-1}\right)\end{array}$ & $0.75 \pm 0.62$ & $0.94 \pm 0.76$ & $<0.001^{d}$ & $3.30 \pm 0.63$ & $3.76 \pm 0.68$ & $<0.001^{d}$ & $0.20 \pm 0.16$ & $0.46 \pm 0.18$ & $<0.001^{d}$ \\
\hline Stride length (m) & $0.89 \pm 0.26$ & $1.01 \pm 0.29$ & $<0.001^{d}$ & $1.60 \pm 0.12$ & $1.64 \pm 0.13$ & $0.002^{d}$ & $0.11 \pm 0.06$ & $0.03 \pm 0.04$ & $<0.001^{d}$ \\
\hline $\begin{array}{l}\text { Cadence (steps per } \\
\text { min) }\end{array}$ & $88.65 \pm 51.45$ & $100.74 \pm 58.92$ & $<0.001^{d}$ & $246.47 \pm 42.66$ & $276.27 \pm 46.81$ & $<0.001^{d}$ & $12.09 \pm 10.91$ & $29.80 \pm 10.90$ & $<0.001^{d}$ \\
\hline $\begin{array}{l}\text { Step length symme- } \\
\text { trye }(\%)\end{array}$ & $82.99 \pm 18.76$ & $83.12 \pm 17.76$ & 0.917 & $96.94 \pm 2.58$ & $97.07 \pm 3.07$ & 0.781 & $0.13 \pm 5.51$ & $0.14 \pm 2.17$ & 0.996 \\
\hline
\end{tabular}

Note: the data are presented using mean \pm s.d.

aThe mean difference between uncued and visuotemporal cue conditions.

${ }^{b} P$-value from the dependent-sample $t$-test with the level of significant difference at $P<0.05$.

${ }^{c} P$-value from the independent-samples $t$-test with the level of significant difference at $P<0.05$.

dIndicates significant difference,
${ }^{\text {e}}$ Calculated using the formula: $\left[\frac{\text { average step length of the more affected leg }}{\text { average step length of the less affected leg }} \times 100\right]$.

Table 3 Walking ability in subjects with incomplete spinal cord injury when they walked with and without a visuotemporal cue

\begin{tabular}{|c|c|c|c|c|c|c|c|c|c|}
\hline \multirow[t]{2}{*}{ Variable } & \multicolumn{3}{|c|}{ Subjects with AIS C $(\mathrm{n}=8)$} & \multicolumn{3}{|c|}{ Subjects with AIS D $(\mathrm{n}=12)$} & \multicolumn{3}{|c|}{ Mean difference $e^{a}$} \\
\hline & $\begin{array}{l}\text { Uncued } \\
\text { condition }\end{array}$ & $\begin{array}{c}\text { Visuotemporal cue } \\
\text { condition }\end{array}$ & $\begin{array}{c}\mathrm{P}- \\
\text { value }^{\mathrm{b}}\end{array}$ & $\begin{array}{l}\text { Uncued } \\
\text { condition }\end{array}$ & $\begin{array}{l}\text { Visuotemporal cue } \\
\text { condition }\end{array}$ & P-value & $\begin{array}{c}\text { Subjects with } \\
\text { AIS C }\end{array}$ & $\begin{array}{c}\text { Subjects with } \\
\text { AIS D }\end{array}$ & $\begin{array}{c}\mathrm{P}- \\
\text { value }^{\mathrm{c}}\end{array}$ \\
\hline $\begin{array}{l}\text { Walking speed } \\
\left(\mathrm{ms}^{-1}\right)\end{array}$ & $0.33 \pm 0.19$ & $0.43 \pm 0.24$ & $0.002^{\mathrm{d}}$ & $1.02 \pm 0.66$ & $1.29 \pm 0.80$ & $<0.001^{d}$ & $0.09 \pm 0.06$ & $0.27 \pm 0.17$ & $0.005^{d}$ \\
\hline Stride length (m) & $0.71 \pm 0.21$ & $0.84 \pm 0.27$ & $0.001^{d}$ & $1.01 \pm 0.22$ & $1.12 \pm 0.25$ & $<0.001^{d}$ & $0.13 \pm 0.07$ & $0.11 \pm 0.04$ & 0.412 \\
\hline $\begin{array}{l}\text { Step length symme- } \\
\operatorname{try}^{\mathrm{e}}(\%)\end{array}$ & $78.68 \pm 24.99$ & $76.57 \pm 24.15$ & 0.365 & $85.87 \pm 13.72$ & $87.49 \pm 11.03$ & 0.258 & $-2.11 \pm 6.16$ & $1.62 \pm 4.71$ & 0.142 \\
\hline
\end{tabular}

Abbreviations: AIS, American Spinal Injury Association (ASIA) Impairment Scale; AIS C, Incomplete. Motor function is preserved below the neurological level, and more than half of key muscles below the neurological level have a muscle grade less than 3; AIS D, Incomplete. Motor function is preserved below the neurological level, and at least half of key muscles below the neurological level have a muscle grade greater than or equal to 3.

level have a muscle grade greater than or equal to
Note: the data are presented using mean \pm s.d.

aThe mean difference between uncued and visuotemporal cue conditions.

${ }^{b} P$-value from the dependent-sample $t$-test with the level of significant difference at $P<0.05$.

${ }^{c} P$-value from the independent-samples $t$-test with the level of significant difference at $P<0.05$.

dIndicates significant difference

${ }^{\text {e}}$ Calculated using the formula: $\left[\frac{\text { average step length of the more affected leg }}{\text { average step length of the less affected leg }} \times 100\right]$.

power of test $(\geqslant 0.90)$ in walking speed and cadence also support the trend, as indicated above, that subjects with less sensorimotor deterioration (AIS D) could increase cadence to enhance walking speed significantly better than those with higher degrees of lesion severity (AIS C). However, the data of stride length and percents of step symmetry had low statistical power $(0.10-0.11)$, they may need a greater number of subjects to provide concrete conclusion.

The findings of this study confirm the benefit of the visuotemporal cue to improve variables associated with walking capacity in subjects with intact brain functions in which the magnitude of effects depends on the degree of internal deterioration of individuals. Nevertheless, some limitations must be kept in mind when interpreting the findings. First, the data were analyzed using the method of manual digitization because it has been reported as a valid and reliable method ${ }^{13}$ that could be executed in a rehabilitation ward. However, a more sophisticated method may be needed to confirm the findings. Second, the time set for the visuotemporal cue relied on the findings of the uncued condition of each subject. Therefore, every subject started the tests with the uncued condition, and the sequence of the tests may affect the findings of the visuotemporal cue condition (due to fatigue or learning effects). Third, the sample size calculation was based on the primary objective and availability of the subjects. Thus the findings of percents of step symmetry (required at least 351 subjects) and some variables in secondary objective showed no significant differences with low statistical power. As the symmetrical step length is an important determinant for the functionality of walking, the reduction of fall risk, and the efficient energy expenditure while walking, ${ }^{19,20}$ a further intervention study using a greater number of subjects with the assessments on its benefit for the retention test and community ambulation, as well as any disadvantages of using the cue, that is, induced fatigue, spasticity or muscle soreness, may thoroughly confirm the utility of the visuotemporal cue in sport and exercise sciences, and rehabilitation treatments. 


\section{DATA ARCHIVING}

There were no data to deposit.

\section{CONFLICT OF INTEREST}

The authors declare no conflict of interest.

\section{ACKNOWLEDGEMENTS}

This study was funded by a grant from Thammasat University, Bangkok, Thailand, and the Improvement of Physical Performance and Quality of Life (IPQ) research group, Khon Kaen University, Khon Kaen, Thailand. We sincerely thank Mr Ian Thomas for his contribution in preparing this manuscript. We also thank Faculty of Associated Medical Sciences, and Graduate School, Khon Kaen University.

1 Field-Fote EC. Spinal cord control of movement: implications for locomotor rehabilitation following spinal cord injury. Phys Ther 2000; 80: 477-484.

2 Lapointe R, Lajoie Y, Serresse O, Barbeau H. Functional community ambulation requirements in incomplete spinal cord injured subjects. Spinal Cord 2001; 39 327-335

3 Van Hedel H. Gait speed in relation to categories of functional ambulation after spinal cord injury. Neurorehabil Neural Repair 2009; 23: 343-350.

4 Amatachaya S, Keawsutthi M, Amatachaya P, Manimmanakorn N. Effects of external cues on gait performance in independent ambulatory incomplete spinal cord injury patients. Spinal Cord 2009; 47: 668-673.

5 Field-Fote E, Tepavac D. Improved intralimb coordination in people with incomplete spinal cord injury following training with body weight support and electrical stimulation. Phys Ther 2002; 82: 707-715.

6 Zorner B, Blanckenhorn WU, Dietz V, Curt A. Clinical algorithm for improved prediction of ambulation and patient stratification after incomplete spinal cord injury. J Neurotrauma 2010; 27: 241-252.
7 Majsak M, Kaminski T, Gentile A, Flanagan J. The reaching movements of patients with Parkinson's disease under self-determined maximal speed and visually cued conditions. Brain 1998; 121: 755-766.

8 Suteerawattananon M, Morrisb G, Etnyrec B, Jankovicd J, Protas E. Effects of visual and auditory cues on gait in individuals with Parkinson's disease. J Neurol Sci 2004 219: 63-69.

9 Thaut M, Leins A, Rice R, Argstatter H, Kenyon G, Mclntosh G et al. Rhythmic auditory stimulation improves gait more than NDT/Bobath training in near-ambulatory patients early poststroke: a single-blind, randomized trial. Clin Rehabil 2007; 21 455-459.

10 Beek P, van Wieringen P. Perspectives on the relation between information and dynamics: an epilogue. Hum Mov Sci 1994; 13: 519-533.

11 Bach-y-Rita P, Kercel S. Sensory substitution and the human-machine interface. Trends Cogn Sci 2003; 7: 541-546.

12 Amatachaya S, Amatachaya P, Keawsutthi M. External cues benefit walking ability of ambulatory patients with spinal cord injury. J Spinal Cord Med 2013; 36: 638-644.

13 Said C, Goldie P, Patla A, Sparrow W. Effect of stroke on step characteristics of obstacle crossing. Arch Phys Med Rehabil 2001; 82: 1712-1719.

14 Kirtley C. The temporal-spatial parameters. In: Clinical Gait Analysis: Theory and Practice. Livingstone: Edinburgh, Scotland, 2006, pp 20-21.

15 Van Hedel H, Dietz V, Curt A. Assessment of walking speed and distance in subjects with an incomplete spinal cord injury. Neurorehabil Neural Repair 2007; 21: 295-301.

16 Gibson J. The Ecological Approach to Visual Perception. Houghton Mifflin company: Boston, MA, USA, 1979.

17 Tanawongsuwan R, Bobick A. Performance analysis of time-distance gait parameters under different speeds. Proceedings of the 4th International Conference on Audio- and Video-Based Biometric Person Authentication; 2003; Guildford, UK, pp 715-724.

18 Capo-Lugo CE, Mullens CH, Brown DA. Maximum walking speeds obtained using treadmill and overground robot system in persons with post-stroke hemiplegia J Neuroeng Rehabil 2012; 9: 80.

19 Teixeira da Cunha Filho I, Lim PA, Qureshy H, Henson H, Monga T, Protas EJ. A comparison of regular rehabilitation and regular rehabilitation with supported treadmill ambulation training for acute stroke patients. J Rehabil Res Dev 2001 38: 245-255.

20 Hausdorff JM, Edelberg HK, Mitchell SL, Goldberger AL, Wei JY. Increased gait unsteadiness in community-dwelling elderly fallers. Arch Phys Med Rehabil 1997; 78 278-283. 